\section{Gehen SMS-Nachrichten an die Nieren?}

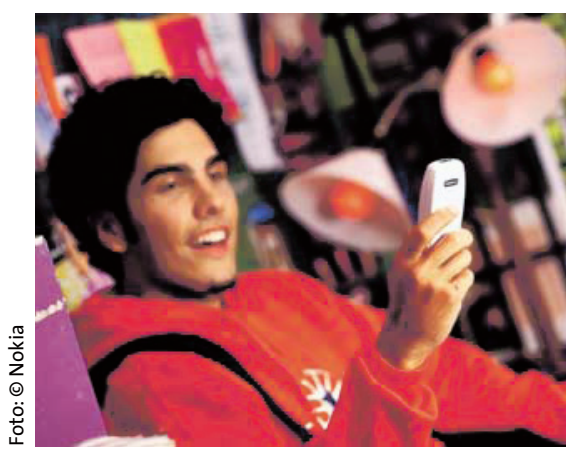

ü ber die möglichen Gesundheitsgefahren bei der Nutzung eines Mobiltelefons gehen die Meinungen nach wie vor auseinander. Die British Medical
Association warnt jetzt sogar davor, dass nicht nur das eigentliche Telefonieren, sondern auch das Senden und Empfangen von SMS-Nachrichten gefährlich sein könnte. Bei der Nutzung des vor allem bei Jugendlichen beliebten "Short Message Service“ wird das Handy etwa auf Taillenhöhe gehalten. Untersuchungen zu den biologischen Effekten der elektromagnetischen Handy-Strahlung in dieser Körperregion fehlten allerdings bisher, bemängeln die britischen Ärzte und befürchten negative Auswirkungen vor allem für die Nieren.

Deutsches Grünes Kreuz

\section{Portale für den Spezialisten}

$\mathrm{N}$ eue Eingangstüren und eine neue Fassade bietet das Internet-Haus Multimedica. Ärzte können sich hier künftig besser orientieren, indem sie ihr spezifisches Fachportal wählen und somit direkt auf für sie relevante Informationen zugreifen. Ein spezielles Allergologie-Portal gibt es bisher nicht. Jedoch stehen dem ALLERGO JOURNAL-Leser eine Reihe von Fachportalen zur Verfügung, darunter Innere Medizin, Dermatologie, Pädiatrie und HNO-Heilkunde. Reinschnuppern und ein Testabo bestellen können Sie unter der Adresse www.multimedica.de/login.

\title{
Juckreiz macht schmerzempfindlich
}

M enschen mit chronischen Hauterkrankungen wie mit atopischem Ekzem reagieren auf Schmerzreize empfindlicher als Gesunde, berichteten Wissenschaftler auf dem Deutschen Schmerzkongress.

Die Experimente der Hautklinik der Berliner Charité und des Psychologischen Instituts der Universität Hamburg ergaben zudem, dass Ekzem-Patienten auf eine Plazebo-Salbe, die Ärzte als schmerzlindernd bezeichnet hatten, besser ansprachen als gesunde Probanden. Durch klassische Konditionierung ließ sich der Effekt steigern: Nach Auf- tragung der Plazebo-Salbe reduzierten die Forscher die Intensität der Schmerzreize. Patienten und Probanden sollten die Salbe mit der Erfahrung „Schmerzlinderung“ verbinden. Dadurch konnten sie die „Wirksamkeit“ des Plazebos verstärken.

Fazit der norddeutschen Ärzte: "Wir sollten den Plazebo-Effekt nicht mehr als zufälliges Anhängsel belächeln, sondern als systematischen Lernprozess zur Steigerung der medikamentösen Effizienz gezielt nutzen."

cl

Deutscher Schmerzkongress, Berlin 2001

\section{Der Arztberuf - Anleitung zum Unglücklichsein?}

W ir ahnten es, jetzt wissen wir's: Glückliche Ärzte sind vom Aussterben bedroht, so (ähnlich) das Ergebnis einer Umfrage des British Medical Journal. An der Umfrage des Magazins nahmen Ärzte aus 90 Ländern teil. Von den 1.540 Beteiligten fühlten sich $41 \%$ unglücklich, nur $17 \%$ glücklich, ebenfalls $17 \%$ sehr unglücklich und weniger als $4 \%$ sehr glücklich.

Die Gründe kennen wir: 62\% der Befragten fühlten sich überarbeitet,
$49 \%$ schlecht bezahlt, $44 \% \mathrm{zu}$ wenig von der Politik unterstützt. Viele Ärzte beklagten zudem, dass sie die Kontrolle über ihre Arbeit verlieren. Ein anderer Punkt betrifft einen kulturellen Aspekt der täglichen Arbeit - die mangelnde Fähigkeit unserer Gesellschaft, mit Schmerz, Krankheit und Tod umzugehen. Viele belastet es zudem, dass die Medien ein zu negatives Bild von Ärzten zeichnen.

Smith R. BMJ 2001; 322: 1073-4

\section{Jedes zweite Asthma vermeidbar?}

W ürde man die Haushaltsallergene und auch die Haustiere eliminieren, könnten etwa 45\% aller Asthmaanfälle bei älteren Kindern und Heranwachsenden vermieden werden, behauptet Bruce Lanphear vom Children's Hospital Medical Center in Cincinnati.

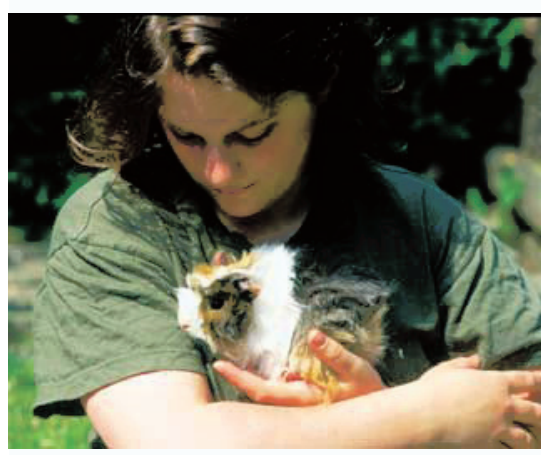

Aus einer Studie an über 5.000 Kindern schließt er, dass mehr als 330.000 von den 4,6 Millionen Asthma-Erkrankungen in den USA auf Haustiere zurückzuführen sind. Für weitere 520.000 Fälle macht er die Hausstaubmilben verantwortlich und 375.000 Fälle seien auf den Kontakt mit Küchenschaben zurückzuführen.

red

Lanphear BP et al. Pediatrics 2001; 107: E98 